\title{
Prediction of Estrogenic Bioactivity of Environmental Chemical Metabolites
}

\section{Supplementary tables and figures}

Pinto et al., 2016

Table S1. Potential negative chemicals for bioactivation.

\begin{tabular}{|c|c|c|c|c|c|}
\hline \multirow[t]{2}{*}{ Parent compound } & \multirow[t]{2}{*}{ CASRN } & \multirow[t]{2}{*}{ SMILES } & \multirow{2}{*}{$\begin{array}{c}\text { Negative } \\
\text { S9 study } \\
\text { (Ref) }\end{array}$} & \multicolumn{2}{|c|}{$\begin{array}{c}\text { Number Uterotrophic } \\
\text { studies }^{*}\end{array}$} \\
\hline & & & & Positive & Negative \\
\hline 2-(2H-Benzotriazol-2-yl)-4-methylphenol (Drometrizole) & $2440-22-4$ & Cc1cc(c(O)cc1)-[n]1[n]c2ccccc2[n]1 & 1,2 & 0 & 2 \\
\hline 2,4-Di-tert-butylphenol & $96-76-4$ & $\mathrm{CC}(\mathrm{C})(\mathrm{C}) \operatorname{c1cc}(\operatorname{ccc} 1 \mathrm{O}) \mathrm{C}(\mathrm{C})(\mathrm{C}) \mathrm{C}$ & 2 & 0 & 1 \\
\hline 2-Ethylhexyl 4-dimethylaminobenzoate (Octyl-dimethyl-PABA) & $21245-02-3$ & $\operatorname{CcccC}(\operatorname{COC}(=0) \operatorname{cocc}(\operatorname{cc} 1) \mathrm{N}(\mathrm{C}) \mathrm{C}) \mathrm{CC}$ & & 0 & 1 \\
\hline 2-Mercaptobenzothiazole & $149-30-4$ & Sc1[n]c2cccce2[s]1 & 2 & 0 & 1 \\
\hline Acrylonitrile & $107-13-1$ & $\mathrm{C}=\mathrm{CC} \# \mathrm{~N}$ & 2 & 0 & 1 \\
\hline Butyl benzyl phthalate & $85-68-7$ & $\operatorname{ccCCOC}(=0) \operatorname{c1ccccc} 1 \mathrm{C}(=0) \mathrm{OCc} 1 \operatorname{ccccc} 1$ & 2 & 0 & 5 \\
\hline Di(2-ethylhexyl) adipate & 103-23-1 & $\operatorname{ccccc}(\operatorname{COC}(=0) \operatorname{ccccC}(=0) \operatorname{OCC}(\operatorname{CcCC}) \operatorname{CC}) \operatorname{CC}$ & 2 & 0 & 4 \\
\hline Di(2-ethylhexyl) phthalate & $117-81-7$ & $\operatorname{ccccC}(\operatorname{COC}(=0) \operatorname{cocccc} 1 \mathrm{C}(=0) \mathrm{OCC}(\operatorname{CCCC}) \mathrm{CC}) \mathrm{CC}$ & 2 & 0 & 6 \\
\hline Dibutyl phthalate & 84-74-2 & $\operatorname{cccCOC}(=0) \operatorname{c1ccccc} 1 \mathrm{C}(=0) \mathrm{OCCCC}$ & 2 & 0 & 9 \\
\hline Dicyclohexyl phthalate & 84-61-7 & $\mathrm{O}=\mathrm{C}(\mathrm{OC} 1 \mathrm{CCCCC} 1) \operatorname{c} 1 \operatorname{ccccc} 1 \mathrm{C}(=\mathrm{O}) \mathrm{OC} 1 \mathrm{CCCCC} 1$ & 2 & 0 & 2 \\
\hline Dioctyl phthalate & $117-84-0$ & $\operatorname{cccccccCOC}(=0) \operatorname{ciccccc} 1 \mathrm{C}(=0) \mathrm{OCCCCCCCC}$ & 2 & 0 & 2 \\
\hline Tetraethylthiuram disulfide (Disulfiram) & $97-77-8$ & $\operatorname{CCN}(\mathrm{CC}) \mathrm{C}(=\mathrm{S}) \mathrm{SSC}(=\mathrm{S}) \mathrm{N}(\mathrm{CC}) \mathrm{CC}$ & 2 & 0 & 1 \\
\hline
\end{tabular}

* Studies obtained from database of rodent uterotrophic assays. ${ }^{3}$ 
Table S2. SMILES notation of parent chemicals and estrogenic metabolites

\begin{tabular}{|c|c|c|c|c|c|}
\hline Parent compound & CASRN & SMILES & Metabolite & CASRN & SMILES \\
\hline $\begin{array}{l}\text { 1-(N-phenylamino) } \\
\text { naphthalene }\end{array}$ & $90-30-2$ & $\operatorname{c1cccc} 2 c(\operatorname{cccc} 21)$ Nc1ccccc1 & ND & & \\
\hline 1,3- Diphenylpropane & $1081-75-0$ & C(CCc1ccccc1)c1ccccc1 & ND & & \\
\hline 2,4-Diphenyl-1-butene & $16606-47-6$ & $\mathrm{C}=\mathrm{C}(\mathrm{CCC} 1=\mathrm{CC}=\mathrm{CC}=\mathrm{C} 1) \mathrm{C} 1=\mathrm{CC}=\mathrm{CC}=\mathrm{C} 1$ & ND & & \\
\hline \multirow{2}{*}{ 2,2-Diphenylpropane } & \multirow{2}{*}{ 778-22-3 } & \multirow{2}{*}{$\mathrm{CC}(\mathrm{C} 1=\mathrm{CC}=\mathrm{CC}=\mathrm{C} 1)(\mathrm{C} 1=\mathrm{CC}=\mathrm{CC}=\mathrm{C} 1) \mathrm{C}$} & $\begin{array}{l}\text { 2-(4-Hydroxyphenyl)-2- } \\
\text { phenylpropane }\end{array}$ & 599-64-4 & $C(C 1=C C=C(0) C=C 1)(C 1 C=C C=C C=1)(C) C$ \\
\hline & & & 4,4'-Dihydroxydiphenylpropane & 80-05-7 & $\mathrm{CC}(\mathrm{C} 1=\mathrm{CC}=\mathrm{C}(\mathrm{O}) \mathrm{C}=\mathrm{C} 1)(\mathrm{C} 1=\mathrm{CC}=\mathrm{C}(0) \mathrm{C}=\mathrm{C} 1) \mathrm{C}$ \\
\hline $\begin{array}{l}\text { 2-Methyl-6-tert- } \\
\text { butylphenol }\end{array}$ & 2219-82-1 & $\operatorname{Cc1cccc}(\operatorname{c1O}) C(C)(C) C$ & ND & & \\
\hline 2-Nitrofluorene & $607-57-8$ & {$[0-][N+](=0) \operatorname{c1cc2} 2 \operatorname{cc} 3 \operatorname{ccccc} 3-\mathrm{c} 2 \mathrm{cc} 1$} & 7-Hydroxy-2-nitrofluorene & $6633-40-5$ & $\begin{array}{l}\mathrm{OC} 1=\mathrm{CC} 2=\mathrm{C}(\mathrm{C}=\mathrm{C} 1) \mathrm{C} 1=\mathrm{C}(\mathrm{C} 2) \mathrm{C}=\mathrm{C}(\mathrm{C}=\mathrm{C} 1)[\mathrm{N}+]([\mathrm{O}- \\
] \mathrm{l})=\mathrm{O}\end{array}$ \\
\hline 4,4'-Dimethoxystilbene & 4705-34-4 & cOc1ccc(cc1)C=Cc1ccc(cc1)OC & ND & & \\
\hline $\begin{array}{l}\text { 4-Tert- } \\
\text { butylphenylsalicylate }\end{array}$ & $87-18-3$ & $\operatorname{cC}(C)(C) \operatorname{c1ccc}(\operatorname{cc} 1) O C(=0) \operatorname{c1ccccc} 10$ & ND & & \\
\hline Azobenzene & 103-33-3 & $\mathrm{N}(=\mathrm{NC} 1=\mathrm{CC}=\mathrm{CC}=\mathrm{C} 1) \mathrm{C} 1=\mathrm{CC}=\mathrm{CC}=\mathrm{C} 1$ & 4-Hydroxyazobenzene & $1689-82-3$ & $\mathrm{C} 1=\mathrm{CC}(\mathrm{O})=\mathrm{CC}=\mathrm{C} 1 / \mathrm{N}=\mathrm{N} / \mathrm{C} 1=\mathrm{CC}=\mathrm{CC}=\mathrm{C} 1$ \\
\hline \multirow{2}{*}{ Benz[a]anthracene } & \multirow{2}{*}{$56-55-3$} & \multirow{2}{*}{$\begin{array}{l}\mathrm{C} 12=\mathrm{CC} 3=\mathrm{C}(\mathrm{C}=\mathrm{CC}=\mathrm{C} 3) \mathrm{C}=\mathrm{C} 1 \mathrm{C}=\mathrm{CC} 1=\mathrm{C} 2 \mathrm{C} \\
=\mathrm{CC}=\mathrm{C} 1\end{array}$} & 3-Hydroxybenz[a]anthracene & 4834-35-9 & $\begin{array}{l}\mathrm{C} 1=\mathrm{C} 2 \mathrm{C}(\mathrm{C} 3=\mathrm{C}(\mathrm{C}=\mathrm{C} 2) \mathrm{C}=\mathrm{C}(\mathrm{O}) \mathrm{C}=\mathrm{C} 3)=\mathrm{CC} 2=\mathrm{C} 1 \mathrm{C}=\mathrm{CC}= \\
\mathrm{C} 2\end{array}$ \\
\hline & & & 4-Hydroxybenz[a]anthracene & $5133-12-0$ & $\mathrm{OC} 1=\mathrm{CC}=\mathrm{CC} 2=\mathrm{C} 1 \mathrm{C}=\mathrm{CC} 1=\mathrm{CC} 3=\mathrm{C}(\mathrm{C}=\mathrm{CC}=\mathrm{C} 3) \mathrm{C}=\mathrm{C} 21$ \\
\hline \multirow{4}{*}{ Benzo[a]pyrene } & \multirow{4}{*}{$50-32-8$} & \multirow{4}{*}{$\begin{array}{l}C 1=C C 2 C 3 C(C=C C=2)=C C=C 2 C=3 C 1=C 1 \\
C(=C 2) C=C C=C 1\end{array}$} & 3-Hydroxybenzo[a]pyrene & $13345-21-6$ & $\begin{array}{l}\mathrm{C} 12=\mathrm{C} 3 \mathrm{C} 4 \mathrm{C}=\mathrm{C} 5 \mathrm{C}(=\mathrm{C} 3 \mathrm{C}=\mathrm{CC} 1=\mathrm{CC}=\mathrm{C}(\mathrm{O}) \mathrm{C} 2=\mathrm{CC}=4) \mathrm{C} \\
=\mathrm{CC}=\mathrm{C} 5\end{array}$ \\
\hline & & & 9-Hydroxybenzo[a]pyrene & $17573-21-6$ & $\begin{array}{l}\mathrm{OC} 1=\mathrm{CC} 2=\mathrm{C}(\mathrm{C}=\mathrm{C} 1) \mathrm{C}=\mathrm{C} 1 \mathrm{C}=\mathrm{CC} 3=\mathrm{CC}=\mathrm{CC} 4=\mathrm{C} 3 \mathrm{C} 1=\mathrm{C} \\
2 \mathrm{C}=\mathrm{C} 4\end{array}$ \\
\hline & & & 8-Hydroxybenzo[a]pyrene & 13345-26-1 & $\begin{array}{l}\mathrm{OC} 1=\mathrm{CC} 2=\mathrm{C}(\mathrm{C}=\mathrm{C} 1) \mathrm{C} 1=\mathrm{CC}=\mathrm{C} 3 \mathrm{C}=\mathrm{CC}=\mathrm{C} 4 \mathrm{C}=\mathrm{CC}(=\mathrm{C} 2) \\
\mathrm{C} 1=\mathrm{C} 34\end{array}$ \\
\hline & & & 7-Hydroxybenzo[a]pyrene & 37994-82-4 & $\begin{array}{l}\mathrm{OC} 1=\mathrm{C} 2 \mathrm{C}=\mathrm{C} 3 \mathrm{C}=\mathrm{CC} 4=\mathrm{CC}=\mathrm{CC} 5=\mathrm{CC}=\mathrm{C}(\mathrm{C} 2=\mathrm{CC}=\mathrm{C} 1) \mathrm{C} \\
3=\mathrm{C} 45\end{array}$ \\
\hline Benzophenone & 119-61-9 & $C 1=C C=C C=C 1 C(=0) C 1=C C=C C=C 1$ & 4-Hydroxybenzophenone & $1137-42-4$ & $C 1(C(=0) C 2=C C=C C=C 2)=C C=C(O) C=C 1$ \\
\hline \multirow{2}{*}{ Benzophenone 3} & \multirow{2}{*}{ 131-57-7 } & \multirow{2}{*}{$\begin{array}{l}\mathrm{C} 1(\mathrm{C}(=) \mathrm{C} 2 \mathrm{C}=\mathrm{CC}=\mathrm{CC}=2) \mathrm{C}(\mathrm{O})=\mathrm{CC}(\mathrm{OC})=\mathrm{C} \\
\mathrm{C}=1\end{array}$} & 2,4-Dihydroxybenzophenone & $131-56-6$ & $C 1(C(=0) C 2 C=C C=C C=2) C(0)=C C(0)=C C=1$ \\
\hline & & & 2,3,4-Trihydroxybenzophenone & $1143-72-2$ & $\mathrm{O}=\mathrm{C}(\mathrm{C} 1=\mathrm{CC}=\mathrm{CC}=\mathrm{C} 1) \mathrm{C} 1=\mathrm{CC}=\mathrm{C}(\mathrm{O}) \mathrm{C}(0)=\mathrm{C} 10$ \\
\hline Biochanin A & $491-80-5$ & $\operatorname{coc} 1 c c c(\operatorname{cc} 1) \mathrm{C} 1=\operatorname{COc} 2 c c(0) \operatorname{cc}(0) \mathrm{c} 2 \mathrm{C} 1=0$ & Genistein & $446-72-0$ & $\operatorname{Oc1cc}(0) \operatorname{cc} 2 \mathrm{OC}=\mathrm{C}(\mathrm{C}(=0) \mathrm{c12}) \mathrm{c1ccc}(\mathrm{O}) \mathrm{cc1}$ \\
\hline \multirow{3}{*}{ Chalcone } & \multirow{3}{*}{$94-41-7$} & \multirow{3}{*}{$\mathrm{O}=\mathrm{C}(\mathrm{C}=\mathrm{Cc} 1 \mathrm{ccccc} 1) \operatorname{c} 1 \operatorname{ccccc} 1$} & 4-Hydroxychalcone & 20426-12-4 & Oc1ccc(cc1)C=CC $(=0) \operatorname{c1ccccc} 1$ \\
\hline & & & 4'-Hydroxychalcone & $2657-25-2$ & Oc1ccc(cc1)C(=O)C=Cc1ccccc1 \\
\hline & & & 2-Hydroxychalcone & 42224-53-3 & $\mathrm{C} 1=\mathrm{CC}=\mathrm{C}(\mathrm{C}=\mathrm{C} 1) \mathrm{C}(=\mathrm{O}) \mathrm{C}=\mathrm{CC} 2=\mathrm{CC}=\mathrm{CC}=\mathrm{C} 2 \mathrm{O}$ \\
\hline \multirow{3}{*}{ Chrysene } & \multirow{3}{*}{ 218-01-9 } & \multirow{3}{*}{$\begin{array}{l}\mathrm{C} 12 \mathrm{C}=\mathrm{CC} 3 \mathrm{C}=\mathrm{CC}=\mathrm{CC}=3 \mathrm{C}=1 \mathrm{C}=\mathrm{CC} 1=\mathrm{C} 2 \mathrm{C}= \\
\mathrm{CC}=\mathrm{C} 1\end{array}$} & 1-Hydroxychrysene & 63019-38-5 & $\mathrm{OC} 1=\mathrm{CC}=\mathrm{CC} 2=\mathrm{C} 1 \mathrm{C}=\mathrm{CC} 1=\mathrm{C} 2 \mathrm{C}=\mathrm{CC} 2=\mathrm{C} 1 \mathrm{C}=\mathrm{CC}=\mathrm{C} 2$ \\
\hline & & & 2-Hydroxychrysene & $65945-06-4$ & $\begin{array}{l}\mathrm{OC} 1=\mathrm{CC} 2=\mathrm{C}(\mathrm{C}=\mathrm{C} 1) \mathrm{C} 1=\mathrm{C}(\mathrm{C}=\mathrm{C} 2) \mathrm{C} 2=\mathrm{C}(\mathrm{C}=\mathrm{CC}=\mathrm{C} 2) \mathrm{C}= \\
\mathrm{C} 1\end{array}$ \\
\hline & & & 3-Hydroxychrysene & $63019-39-6$ & $\begin{array}{l}\mathrm{C} 12 \mathrm{C}=\mathrm{CC} 3=\mathrm{C}(\mathrm{C}=\mathrm{CC}=\mathrm{C} 3) \mathrm{C}=1 \mathrm{C}=\mathrm{CC} 1=\mathrm{C} 2 \mathrm{C}=\mathrm{C}(\mathrm{C}=\mathrm{C} 1) \\
\mathrm{O}\end{array}$ \\
\hline Cypermethrin & $52315-07-8$ & $\begin{array}{l}\mathrm{C} 1(\mathrm{C}(/ \mathrm{C}=\mathrm{C}(/ \mathrm{Cl}) \mathrm{Cl}) \mathrm{C} 1 \mathrm{C}(\mathrm{OC}(\mathrm{C} \text { \#N)C1C=C(O)} \\
\mathrm{C2}=\mathrm{CC}=\mathrm{CC}=\mathrm{C} 2) \mathrm{C}=\mathrm{CC}=1)=0)(\mathrm{C}) \mathrm{C}\end{array}$ & 3-Phenoxybenzaldehyde & $39515-51-0$ & $C(=0) C 1=C C(O C 2=C C=C C=C 2)=C C=C 1$ \\
\hline Dibenzyl & 103-29-7 & $\mathrm{C}(\mathrm{C}$ c1ccccc1)c1ccccc1 & 4,4'-Dihydroxydibenzyl & 6052-84-2 & c1(0)ccc(cc1)CCc2ccc(0)cc2 \\
\hline \multirow{3}{*}{ Diphenyl } & \multirow{3}{*}{$92-52-4$} & \multirow{3}{*}{$\mathrm{C}_{1}=\mathrm{C}(\mathrm{C} 2=\mathrm{CC}=\mathrm{CC}=\mathrm{C} 2) \mathrm{C}=\mathrm{CC}=\mathrm{C} 1$} & 4-Hydroxydiphenyl & $92-69-3$ & $\mathrm{OC} 1=\mathrm{CC}=\mathrm{C}(\mathrm{C} 2=\mathrm{CC}=\mathrm{CC}=\mathrm{C} 2) \mathrm{C}=\mathrm{C} 1$ \\
\hline & & & 3-Hydroxydiphenyl & 580-51-8 & $\mathrm{C} 1(=\mathrm{CC}(0)=\mathrm{CC}=\mathrm{C} 1) \mathrm{C} 1=\mathrm{CC}=\mathrm{CC}=\mathrm{C} 1$ \\
\hline & & & 4,4'-Dihydroxydiphenyl & $92-88-6$ & $\mathrm{C} 1(\mathrm{C} 2=\mathrm{CC}=\mathrm{C}(0) \mathrm{C}=\mathrm{C} 2)=\mathrm{CC}=\mathrm{C}(0) \mathrm{C}=\mathrm{C} 1$ \\
\hline
\end{tabular}




\begin{tabular}{|c|c|c|c|c|c|}
\hline Diphenylacetylene & 501-65-5 & $\operatorname{c1ccccc1C\# Cc1ccccc1}$ & ND & & \\
\hline \multirow{2}{*}{ Diphenylmethane } & \multirow{2}{*}{ 101-81-5 } & \multirow{2}{*}{$C(C 1 C=C C=C C=1) C 1=C C=C C=C 1$} & 4-Hydroxydiphenylmethane & 101-53-1 & $C 1(0)=C C=C(C C 2=C C=C C=C 2) C=C 1$ \\
\hline & & & 4,4'-Dihydroxydiphenylmethane & $620-92-8$ & $\mathrm{C} 1(\mathrm{CC} 2 \mathrm{C}=\mathrm{CC}(0)=\mathrm{CC}=2) \mathrm{C}=\mathrm{CC}(0)=\mathrm{CC}=1$ \\
\hline Fluoranthene & 206-44-0 & $\begin{array}{l}\mathrm{C} 12 \mathrm{C} 3 \mathrm{C} 4 \mathrm{C}=\mathrm{CC}=\mathrm{CC}=4 \mathrm{C}=1 \mathrm{C}=\mathrm{CC}=\mathrm{C} 2 \mathrm{C}=\mathrm{CC} \\
=3\end{array}$ & 3-Hydroxyfluoranthene & 17798-09-3 & $\mathrm{OC} 1=\mathrm{C} 2 \mathrm{C}=\mathrm{CC}=\mathrm{C} 3 \mathrm{C} 4=\mathrm{CC}=\mathrm{CC}=\mathrm{C} 4 \mathrm{C}(\mathrm{C}=\mathrm{C} 1)=\mathrm{C} 23$ \\
\hline Fluorene & 86-73-7 & $\mathrm{C} 12 \mathrm{C} 3=\mathrm{C}(\mathrm{C}=\mathrm{CC}=\mathrm{C} 3) \mathrm{CC}=1 \mathrm{C}=\mathrm{CC}=\mathrm{C} 2$ & 2-Hydroxyfluorene & 2443-58-5 & $C 12 C 3=C(C=C C=C 3) C C=1 C=C(0) C=C 2$ \\
\hline Formonometin & $485-72-3$ & $\operatorname{coc} 1 \mathrm{ccc}(\mathrm{cc} 1) \mathrm{C} 1=\operatorname{COc} 2 \mathrm{cc}(0) \operatorname{ccc} 2 \mathrm{C} 1=0$ & Daidzein & $486-66-8$ & Oc1ccc(cc1)C1=COc2cc(0)ccc2C1=0 \\
\hline Mestranol & 72-33-3 & $\begin{array}{l}\text { CC12CCC3C(CCc4cc(ccc43)OC)C1CCC2( } \\
\text { O)C\#C }\end{array}$ & 17a-eth ynylestradiol & $57-63-6$ & $\operatorname{cc} 12 \mathrm{CcC} 3 \mathrm{C}(\mathrm{CCc} 4 \mathrm{cc}(0) \operatorname{ccc} 43) \mathrm{C} 1 \mathrm{CcC} 2(0) \mathrm{C \# C}$ \\
\hline \multirow{2}{*}{ Methoxychlor } & \multirow{2}{*}{ 72-43-5 } & \multirow{2}{*}{$\begin{array}{l}\mathrm{ClC}(\mathrm{C}(\mathrm{C} 1=\mathrm{CC}=\mathrm{C}(\mathrm{OC}) \mathrm{C}=\mathrm{C} 1) \mathrm{C} 1=\mathrm{CC}=\mathrm{C}(\mathrm{OC}) \\
\mathrm{C}=\mathrm{C} 1)(\mathrm{Cl}) \mathrm{Cl}\end{array}$} & Mono-hydroxymethoxychlor & 28463-03-8 & $\begin{array}{l}\mathrm{C}(\mathrm{C}(\mathrm{C} 1=\mathrm{CC}=\mathrm{C}(\mathrm{OC}) \mathrm{C}=\mathrm{C} 1) \mathrm{C} 1 \mathrm{C}=\mathrm{CC}(0)=\mathrm{CC}=1)(\mathrm{Cl})(\mathrm{Cl}) \\
\mathrm{Cl}\end{array}$ \\
\hline & & & HPTE & 2971-36-0 & $\mathrm{C}(\mathrm{C}(\mathrm{C} 1 \mathrm{C}=\mathrm{CC}(\mathrm{O})=\mathrm{CC}=1) \mathrm{C} 1 \mathrm{C}=\mathrm{CC}(\mathrm{O})=\mathrm{CC}=1)(\mathrm{Cl})(\mathrm{Cl}) \mathrm{Cl}$ \\
\hline \multirow{2}{*}{ Naphthalene } & \multirow{2}{*}{$91-20-3$} & \multirow{2}{*}{$\mathrm{C} 1=\mathrm{C} 2 \mathrm{C}(\mathrm{C}=\mathrm{CC}=\mathrm{C} 2)=\mathrm{CC}=\mathrm{C} 1$} & 1-Naphthol & $90-15-3$ & $\mathrm{OC} 1=\mathrm{CC}=\mathrm{CC} 2 \mathrm{C} 1=\mathrm{CC}=\mathrm{CC}=2$ \\
\hline & & & 2-Naphthol & 135-19-3 & $\mathrm{C} 12 \mathrm{C}=\mathrm{CC}=\mathrm{CC}=1 \mathrm{C}=\mathrm{CC}(\mathrm{O})=\mathrm{C} 2$ \\
\hline Nonylphenol ethoxylate & 26027-38-3 & $\operatorname{ccccccccC} 1 \operatorname{ccc}(\operatorname{cc} 1) 0 \operatorname{ccocc0}$ & ND & & \\
\hline o-Benzylphenol & 28994-41-4 & Oc1ccccc1Cc1ccccc1 & ND & & \\
\hline Ortho-phenylphenol & $90-43-7$ & Oc1ccccc1-c1ccccc1 & ND & & \\
\hline \multirow{3}{*}{ Permethrin } & \multirow{3}{*}{$52645-53-1$} & \multirow{3}{*}{$\begin{array}{l}\mathrm{C} 1=\mathrm{CC}=\mathrm{C}(\mathrm{OC} 2=\mathrm{CC}=\mathrm{CC}=\mathrm{C} 2) \mathrm{C}=\mathrm{C} 1 \mathrm{COC}(=\mathrm{O} \\
) \mathrm{C} 1 \mathrm{C}(\mathrm{C})(\mathrm{C}) \mathrm{C} 1 / \mathrm{C}=\mathrm{C}(/ \mathrm{Cl}) \mathrm{Cl}\end{array}$} & 3-Phenoxybenzylalcohol & 13826-35-2 & $\mathrm{C} 1(=\mathrm{CC}=\mathrm{CC}(\mathrm{OC} 2=\mathrm{CC}=\mathrm{CC}=\mathrm{C} 2)=\mathrm{C} 1) \mathrm{CO}$ \\
\hline & & & $\begin{array}{l}\text { 3-(4'-Hydroxyphenoxy)- } \\
\text { benzylalcohol }\end{array}$ & 63987-19-9 & $O C C 1=C C=C C(O C 2=C C=C(O) C=C 2)=C 1$ \\
\hline & & & 3-Phenoxybenzaldehyde & $39515-51-0$ & $C(=0) C 1=C C(O C 2=C C=C C=C 2)=C C=C 1$ \\
\hline \multirow{2}{*}{ Phenanthrene } & \multirow{2}{*}{ 85-01-8 } & \multirow{2}{*}{$C 12 C 3=C(C=C C=C 3) C=C C=1 C=C C=C 2$} & 2-Phenanthrol & $605-55-0$ & $O C 1=C C 2=C(C=C 1) C 1=C(C=C C=C 1) C=C 2$ \\
\hline & & & 9-Phenanthrol & 484-17-3 & $\mathrm{C} 12=\mathrm{CC}=\mathrm{CC}=\mathrm{C} 1 \mathrm{C}(\mathrm{O})=\mathrm{CC} 1=\mathrm{C} 2 \mathrm{C}=\mathrm{CC}=\mathrm{C} 1$ \\
\hline Pyrene & 129-00-0 & $\begin{array}{l}\mathrm{C} 12 \mathrm{C} 3 \mathrm{C} 4=\mathrm{CC}=\mathrm{CC}=3 \mathrm{C}=\mathrm{CC}=1 \mathrm{C}=\mathrm{CC}=\mathrm{C} 2 \mathrm{C}= \\
\mathrm{C} 4\end{array}$ & 1-Hydroxypyrene & $5315-79-7$ & $C 12=C 3 C 4 C=C C(=C 3 C=C C 1=C C=C C 2=C C=4) O$ \\
\hline \multirow{2}{*}{ Trans-stilbene } & \multirow{2}{*}{ 103-30-0 } & \multirow{2}{*}{$\mathrm{C} 1(=\mathrm{CC}=\mathrm{CC}=\mathrm{C} 1) / \mathrm{C}=\mathrm{C} / \mathrm{C} 1=\mathrm{CC}=\mathrm{CC}=\mathrm{C} 1$} & Trans-4-hydroxystilbene & $6554-98-9$ & $\mathrm{C}(=\mathrm{C} / \mathrm{C} 1=\mathrm{CC}=\mathrm{CC}=\mathrm{C} 1) \mid \mathrm{C} 1 \mathrm{C}=\mathrm{CC}(0)=\mathrm{CC}=1$ \\
\hline & & & Trans-4,4'-dihydroxystilbene & $659-22-3$ & $\mathrm{C}(=\mathrm{C} / \mathrm{C} 1=\mathrm{CC}=\mathrm{C}(\mathrm{O}) \mathrm{C}=\mathrm{C} 1) \backslash \mathrm{C} 1=\mathrm{CC}=\mathrm{C}(0) \mathrm{C}=\mathrm{C} 1$ \\
\hline $\begin{array}{l}\text { Trans-1,2- } \\
\text { diphenylcyclobutane }\end{array}$ & 20071-09-4 & $\mathrm{C} 1 \mathrm{CC}(\mathrm{C} 1 \mathrm{c} 1 \mathrm{ccccc} 1) \operatorname{c1ccccc} 1$ & $\begin{array}{l}\text { Trans-1-(4-hydroxyphenyl)-2- } \\
\text { phenylcylobutane }\end{array}$ & & $\operatorname{c1}(0) \operatorname{ccc}(\operatorname{cc} 1) \mathrm{C} 2 \mathrm{C}(\mathrm{c} 3 \operatorname{ccccc} 3) \mathrm{cc} 2$ \\
\hline $\begin{array}{l}\text { Trans-4-phen yl-3-buten- } \\
\text { 2-one }\end{array}$ & $1896-62-4$ & $\mathrm{CC}(=\mathrm{O}) \mathrm{C}=\mathrm{CC} 1=\mathrm{CC}=\mathrm{CC}=\mathrm{C} 1$ & $\begin{array}{l}\text { Trans-4-(4-hydroxyphenyl)-3-buten- } \\
\text { 2-one }\end{array}$ & $3160-35-8$ & $C C(=0) C=C C 1=C C=C(0) C=C 1$ \\
\hline Trans-stilbene oxide & 1439-07-2 & $\mathrm{C} 1=\mathrm{CC}=\mathrm{C}(\mathrm{C}=\mathrm{C} 1) \mathrm{C} 2 \mathrm{C}(\mathrm{O} 2) \mathrm{C} 3=\mathrm{CC}=\mathrm{CC}=\mathrm{C} 3$ & ND & & \\
\hline Trans-a-methylstilbene & $833-81-8$ & $\mathrm{CC}(=\mathrm{CC} 1=\mathrm{CC}=\mathrm{CC}=\mathrm{C} 1) \mathrm{C} 2=\mathrm{CC}=\mathrm{CC}=\mathrm{C} 2$ & 4,4'-Dihydroxy-a-methylstilbene & $72108-22-6$ & $\mathrm{C}(/ \operatorname{c} 1 \mathrm{ccc}(0) \operatorname{cc} 1)(=\mathrm{C} \backslash \mathrm{c} 2 \operatorname{ccc}(0) \operatorname{cc} 2) \backslash \mathrm{C}$ \\
\hline
\end{tabular}

ND - not determined 
Table S3. Uterotrophic studies for parent chemicals and metabolites

\begin{tabular}{|c|c|c|c|c|c|c|c|c|c|c|c|}
\hline \multirow[t]{2}{*}{ Parent compound } & \multirow[t]{2}{*}{ CASRN } & \multicolumn{3}{|c|}{ Uterotrophic (UT) studies } & \multirow[t]{2}{*}{ Ref } & \multirow[t]{2}{*}{ Estrogenic Metabolite } & \multirow[t]{2}{*}{ CASRN } & \multicolumn{3}{|c|}{ Uterotrophic (UT) studies } & \multirow[t]{2}{*}{ Ref } \\
\hline & & $\begin{array}{l}\text { \# UT } \\
\text { studies }\end{array}$ & Positive & Negative & & & & $\begin{array}{l}\text { \# UT } \\
\text { studies }\end{array}$ & Positive & Negative & \\
\hline $\begin{array}{l}\text { 1-(N-phenylamino) } \\
\text { naphthalene }\end{array}$ & $90-30-2$ & 0 & & & & ND & & & & & \\
\hline 1,3- Diphenylpropane & $1081-75-0$ & 0 & & & & ND & & & & & \\
\hline $\begin{array}{l}\text { 2,4-Diphenyl-1- } \\
\text { butene }\end{array}$ & $16606-47-6$ & 1 & 0 & 1 & 4 & ND & & & & & \\
\hline \multirow[t]{2}{*}{ 2,2-Diphenylpropane } & \multirow[t]{2}{*}{$778-22-3$} & \multirow[t]{2}{*}{0} & & & & 2-(4-Hydroxyphenyl)-2-phenylpropane & 599-64-4 & 2 & 2 & 0 & 3 \\
\hline & & & & & & 4,4'-Dihydroxydiphenylpropane & $80-05-7$ & 121 & 83 & 38 & 3 \\
\hline $\begin{array}{l}\text { 2-Methyl-6-tert- } \\
\text { butylphenol }\end{array}$ & $2219-82-1$ & 0 & & & & ND & & & & & \\
\hline 2-Nitrofluorene & $607-57-8$ & 0 & & & & 7-Hydroxy-2-nitrofluorene & $6633-40-5$ & 0 & & & \\
\hline $\begin{array}{l}4,4^{\prime}- \\
\text { Dimethoxystilbene }\end{array}$ & $4705-34-4$ & 0 & & & & ND & & & & & \\
\hline $\begin{array}{l}\text { 4-Tert- } \\
\text { butylphenylsalicylate }\end{array}$ & 87-18-3 & 0 & & & & ND & & & & & \\
\hline Azobenzene & 103-33-3 & 0 & & & & 4-Hydroxyazobenzene & $1689-82-3$ & 2 & 2 & 0 & 5,6 \\
\hline \multirow[t]{2}{*}{ Benz[a]anthracene } & \multirow[t]{2}{*}{$56-55-3$} & \multirow[t]{2}{*}{4} & \multirow[t]{2}{*}{1} & \multirow[t]{2}{*}{3} & 3 & 3-Hydroxybenz[a]anthracene & $4834-35-9$ & 0 & & & \\
\hline & & & & & & 4-Hydroxybenz[a]anthracene & $5133-12-0$ & 0 & & & \\
\hline \multirow[t]{4}{*}{ Benzo[a]pyrene } & \multirow[t]{4}{*}{$50-32-8$} & \multirow[t]{4}{*}{3} & \multirow[t]{4}{*}{1} & \multirow[t]{4}{*}{2} & \multirow[t]{4}{*}{1,8} & 3-Hydroxybenzo[a]pyrene & $13345-21-6$ & 2 & 0 & 2 & g \\
\hline & & & & & & 9-Hydroxybenzo[a]pyrene ${ }^{\dagger}$ & $17573-21-6$ & 2 & 1 & 1 & g \\
\hline & & & & & & 8-Hydroxybenzo[a]pyrene & $13345-26-1$ & 0 & & & \\
\hline & & & & & & 7-Hydroxybenzo[a]pyrene & $37994-82-4$ & 0 & & & \\
\hline Benzophenone & 119-61-9 & 6 & 3 & 3 & 3 & 4-Hydroxybenzophenone & $1137-42-4$ & 3 & 3 & 0 & $\begin{array}{c}5,6 \\
10\end{array}$ \\
\hline \multirow[t]{2}{*}{ Benzophenone 3} & \multirow[t]{2}{*}{$131-57-7$} & \multirow[t]{2}{*}{5} & \multirow[t]{2}{*}{1} & \multirow[t]{2}{*}{4} & 3 & 2,4-Dihydroxybenzophenone & $131-56-6$ & 6 & 6 & 0 & $\begin{array}{l}5,1 \\
11-13\end{array}$ \\
\hline & & & & & & 2,3,4-Trihydroxybenzophenone & $1143-72-2$ & 1 & 1 & 0 & 5 \\
\hline Biochanin A & $491-80-5$ & 6 & 4 & 2 & 3 & Genistein & $446-72-0$ & 101 & 83 & 18 & 3 \\
\hline \multirow[t]{3}{*}{ Chalcone } & \multirow[t]{3}{*}{$94-41-7$} & 0 & & & & 4-Hydroxychalcone & $20426-12-4$ & 0 & & & \\
\hline & & & & & & 4'-Hydroxychalcone & $2657-25-2$ & 0 & & & \\
\hline & & & & & & 2-Hydroxychalcone & $42224-53-3$ & 0 & & & \\
\hline Chrysene & 218-01-9 & 0 & & & & 1-Hydroxychrysene & 63019-38-5 & 0 & & & \\
\hline & & & & & & 2-Hydroxychrysene & $65945-06-4$ & 0 & & & \\
\hline & & & & & & 3-Hydroxychrysene & 63019-39-6 & 0 & & & \\
\hline
\end{tabular}




\begin{tabular}{|c|c|c|c|c|c|c|c|c|c|c|c|}
\hline Cypermethrin & $52315-07-8$ & 2 & 1 & 1 & 3 & 3-Phenoxybenzaldehyde & $39515-51-0$ & 0 & & & \\
\hline Dibenzyl & $103-29-7$ & 0 & & & & 4,4'-Dihydroxydibenzyl & $6052-84-2$ & 0 & & & \\
\hline \multirow[t]{3}{*}{ Diphenyl } & \multirow[t]{3}{*}{$92-52-4$} & \multirow[t]{3}{*}{1} & \multirow[t]{3}{*}{0} & \multirow[t]{3}{*}{1} & \multirow[t]{3}{*}{3} & 4-Hydroxydiphenyl & $92-69-3$ & 1 & 1 & 0 & 3 \\
\hline & & & & & & 3-Hydroxydiphenyl & $580-51-8$ & 0 & & & \\
\hline & & & & & & 4,4'-Dihydroxydiphenyl & $92-88-6$ & 2 & 2 & 0 & 5,13 \\
\hline Diphenylacetylene & $501-65-5$ & 0 & - & - & - & ND & & & & & \\
\hline \multirow[t]{2}{*}{ Diphenylmethane } & \multirow[t]{2}{*}{$101-81-5$} & \multirow[t]{2}{*}{0} & \multirow[t]{2}{*}{-} & \multirow[t]{2}{*}{-} & \multirow[t]{2}{*}{ - } & 4-Hydroxydiphenylmethane & $101-53-1$ & 1 & 1 & 0 & 6 \\
\hline & & & & & & 4,4'-Dihydroxydiphenylmethane & $620-92-8$ & 2 & 2 & 0 & 5,13 \\
\hline Fluoranthene & $206-44-0$ & 1 & 1 & 0 & 3 & 3-Hydroxyfluoranthene & $17798-09-3$ & 0 & & & \\
\hline Fluorene & $86-73-7$ & 0 & - & - & - & 2-Hydroxyfluorene & $2443-58-5$ & 0 & & & \\
\hline Formonometin & $485-72-3$ & 1 & 1 & 0 & 14 & Daidzein & $486-66-8$ & 10 & 5 & 5 & 3 \\
\hline Mestranol & $72-33-3$ & 14 & 14 & 0 & 3 & 17a-Ethynylestradiol & $57-63-6$ & 216 & 206 & 10 & 3 \\
\hline \multirow[t]{2}{*}{ Methoxychlor } & \multirow[t]{2}{*}{$72-43-5$} & \multirow[t]{2}{*}{64} & \multirow[t]{2}{*}{53} & \multirow[t]{2}{*}{11} & \multirow[t]{2}{*}{3} & Mono-Hydroxymethoxychlor & $28463-03-8$ & 0 & & & \\
\hline & & & & & & HPTE & $2971-36-0$ & 5 & 5 & 0 & 3 \\
\hline \multirow[t]{2}{*}{ Naphthalene } & \multirow[t]{2}{*}{$91-20-3$} & \multirow[t]{2}{*}{0} & \multirow[t]{2}{*}{-} & \multirow[t]{2}{*}{-} & \multirow[t]{2}{*}{ - } & 1-Naphthol & $90-15-3$ & 0 & & & \\
\hline & & & & & & 2-Naphthol & $135-19-3$ & 1 & 0 & 1 & 3 \\
\hline $\begin{array}{l}\text { Nonylphenol } \\
\text { ethoxylate }\end{array}$ & $26027-38-3$ & 0 & - & - & - & ND & & & & & \\
\hline ortho-Benzylphenol & $28994-41-4$ & 0 & - & - & - & ND & & & & & \\
\hline ortho-Phenylphenol & $90-43-7$ & 1 & 0 & 1 & 15 & ND & & & & & \\
\hline \multirow[t]{3}{*}{ Permethrin } & \multirow[t]{3}{*}{$52645-53-1$} & \multirow[t]{3}{*}{2} & \multirow[t]{3}{*}{1} & 1 & 3 & 3-Phenoxybenzylalcohol & $13826-35-2$ & 0 & & & \\
\hline & & & & & & 3-(4'-Hydroxyphenoxy)-benzylalcohol & $63987-19-9$ & 1 & 0 & 1 & 3 \\
\hline & & & & & & 3-Phenoxybenzaldehyde & $39515-51-0$ & 0 & & & \\
\hline Phenanthrene & $85-01-8$ & 0 & - & - & - & 2-Phenanthrol & $605-55-0$ & 0 & & & \\
\hline & & & & & & 9-Phenanthrol & $484-17-3$ & 0 & & & \\
\hline Pyrene & $129-00-0$ & 0 & - & - & - & 1-Hydroxypyrene & $5315-79-7$ & 0 & & & \\
\hline Trans-stilbene & $103-30-0$ & 1 & 1 & 0 & 16 & Trans-4-Hydroxystilbene & $6554-98-9$ & 1 & 1 & 0 & 16 \\
\hline & & & & & & Trans-4,4'-Dihydroxystilbene & $659-22-3$ & 1 & 1 & 0 & 16 \\
\hline $\begin{array}{l}\text { Trans-1,2- } \\
\text { diphenylcyclobutane }\end{array}$ & $20071-09-4$ & 1 & 0 & 1 & 4 & $\begin{array}{l}\text { Trans-1-(4-hydroxyphenyl)-2- } \\
\text { phenylcylobutane }\end{array}$ & & 0 & & & \\
\hline $\begin{array}{l}\text { Trans-4-phenyl-3- } \\
\text { buten-2-one }\end{array}$ & $1896-62-4$ & 0 & - & - & - & $\begin{array}{l}\text { Trans-4-(4-hydroxyphenyl)-3-buten-2- } \\
\text { one }\end{array}$ & $3160-35-8$ & 0 & & & \\
\hline Trans-stilbene oxide & $1439-07-2$ & 0 & - & - & - & ND & & & & & \\
\hline $\begin{array}{l}\text { Trans- } \alpha- \\
\text { methylstilbene }\end{array}$ & $833-81-8$ & 0 & - & - & - & 4,4'-Dihydroxy-a-methylstilbene & $72108-22-6$ & 0 & & & \\
\hline
\end{tabular}

ND: not determined; $\uparrow:$ increase in uterine weight at three doses tested; not considered relevant by the authors. 
Table S4. Averaged ER QSAR scores for predicted primary and secondary sets of metabolites for negative chemicals ${ }^{a}$

\begin{tabular}{|c|c|c|c|c|c|c|c|c|c|c|c|c|c|c|c|c|c|c|}
\hline \multirow{3}{*}{ Chemical } & \multirow{2}{*}{\multicolumn{3}{|c|}{ OCHEM }} & \multirow{2}{*}{\multicolumn{3}{|c|}{ LM }} & \multirow{2}{*}{\multicolumn{3}{|c|}{ Unistra }} & \multicolumn{9}{|c|}{ OCHEM, LM \& Unistra } \\
\hline & & & & & & & & & & \multicolumn{3}{|c|}{$\mathbf{P}$} & \multicolumn{3}{|c|}{ M1 } & \multicolumn{3}{|c|}{ M2 } \\
\hline & $\mathbf{P}$ & M1 & M2 & $\mathbf{P}$ & M1 & M2 & $\mathbf{P}$ & M1 & M2 & Avg & St Dev & $\begin{array}{c}\text { AC50 } \\
(\mu \mathrm{M})\end{array}$ & Avg & $\begin{array}{l}\text { St } \\
\text { Dev }\end{array}$ & $\begin{array}{c}\text { AC50 } \\
(\mu \mathrm{M})\end{array}$ & Avg & St Dev & $\begin{array}{c}\text { AC50 } \\
(\mu \mathrm{M})\end{array}$ \\
\hline Acrylonitrile & 0.004 & 0.006 & 0.006 & 0.005 & 0.004 & 0.005 & 0.000 & 0.000 & 0.000 & 0.003 & 0.003 & 4867.73 & 0.003 & 0.003 & 4867.73 & 0.004 & 0.003 & 4135.04 \\
\hline Disulfiram & 0.000 & 0.016 & 0.016 & 0.000 & 0.000 & 0.004 & 0.000 & 0.000 & 0.000 & 0.000 & 0.000 & 0.00 & 0.005 & 0.009 & 3402.35 & 0.007 & 0.008 & 1986.75 \\
\hline Di(2-ethylhexyl) adipate & 0.000 & 0.001 & 0.009 & 0.001 & 0.001 & 0.004 & 0.030 & 0.030 & 0.052 & 0.010 & 0.017 & 1524.71 & 0.011 & 0.017 & 1370.70 & 0.021 & 0.026 & 646.95 \\
\hline Dioctyl phthalate & 0.000 & 0.001 & - & 0.006 & 0.003 & - & 0.100 & 0.100 & - & 0.035 & 0.056 & 400.76 & 0.035 & 0.056 & 400.76 & - & - & - \\
\hline Dibutyl phthalate & 0.006 & 0.012 & 0.022 & 0.006 & 0.013 & 0.041 & 0.070 & 0.070 & 0.075 & 0.028 & 0.037 & 472.46 & 0.031 & 0.033 & 441.73 & 0.046 & 0.027 & 288.10 \\
\hline Drometrizole & 0.057 & 0.048 & 0.034 & 0.063 & 0.081 & 0.083 & 0.020 & 0.020 & 0.031 & 0.047 & 0.023 & 277.86 & 0.050 & 0.030 & 247.13 & 0.049 & 0.029 & 257.37 \\
\hline 2-Mercaptobenzothiazole & 0.000 & 0.006 & 0.008 & 0.006 & 0.131 & 0.135 & 0.000 & 0.000 & 0.011 & 0.002 & 0.003 & 7925.75 & 0.046 & 0.074 & 288.10 & 0.051 & 0.072 & 236.89 \\
\hline 2,4-Di-tert-butylphenol & 0.024 & 0.015 & 0.017 & 0.000 & 0.082 & 0.100 & 0.080 & 0.080 & 0.079 & 0.035 & 0.041 & 400.76 & 0.059 & 0.038 & 187.68 & 0.065 & 0.043 & 170.88 \\
\hline Dicyclohexyl phthalate & 0.035 & 0.023 & 0.031 & 0.084 & 0.073 & 0.076 & 0.080 & 0.080 & 0.097 & 0.066 & 0.027 & 168.08 & 0.059 & 0.031 & 187.68 & 0.068 & 0.034 & 162.48 \\
\hline Butylbenzyl phthalate & 0.052 & 0.042 & 0.044 & 0.068 & 0.086 & 0.161 & 0.070 & 0.070 & 0.087 & 0.064 & 0.010 & 173.68 & 0.066 & 0.022 & 168.08 & 0.097 & 0.059 & 92.97 \\
\hline Di(2-ethylhexyl) phthalate & 0.004 & 0.001 & 0.012 & 0.308 & 0.271 & 0.214 & 0.090 & 0.090 & 0.103 & 0.134 & 0.157 & 54.12 & 0.121 & 0.137 & 67.77 & 0.110 & 0.101 & 79.32 \\
\hline Octyl-dimethyl-PABA & 0.001 & 0.038 & 0.042 & 0.333 & 0.344 & 0.349 & 0.020 & 0.054 & 0.062 & 0.118 & 0.186 & 70.92 & 0.145 & 0.172 & 47.24 & 0.151 & 0.172 & 44.90 \\
\hline
\end{tabular}

${ }^{a}$ The mean of ER QSAR scores for all putative primary and secondary metabolites were calculated for individual models, and the results were averaged in order to obtain a prediction of estrogenicity for parent chemicals, primary and secondary metabolites combining the outputs from the three QSAR models. 


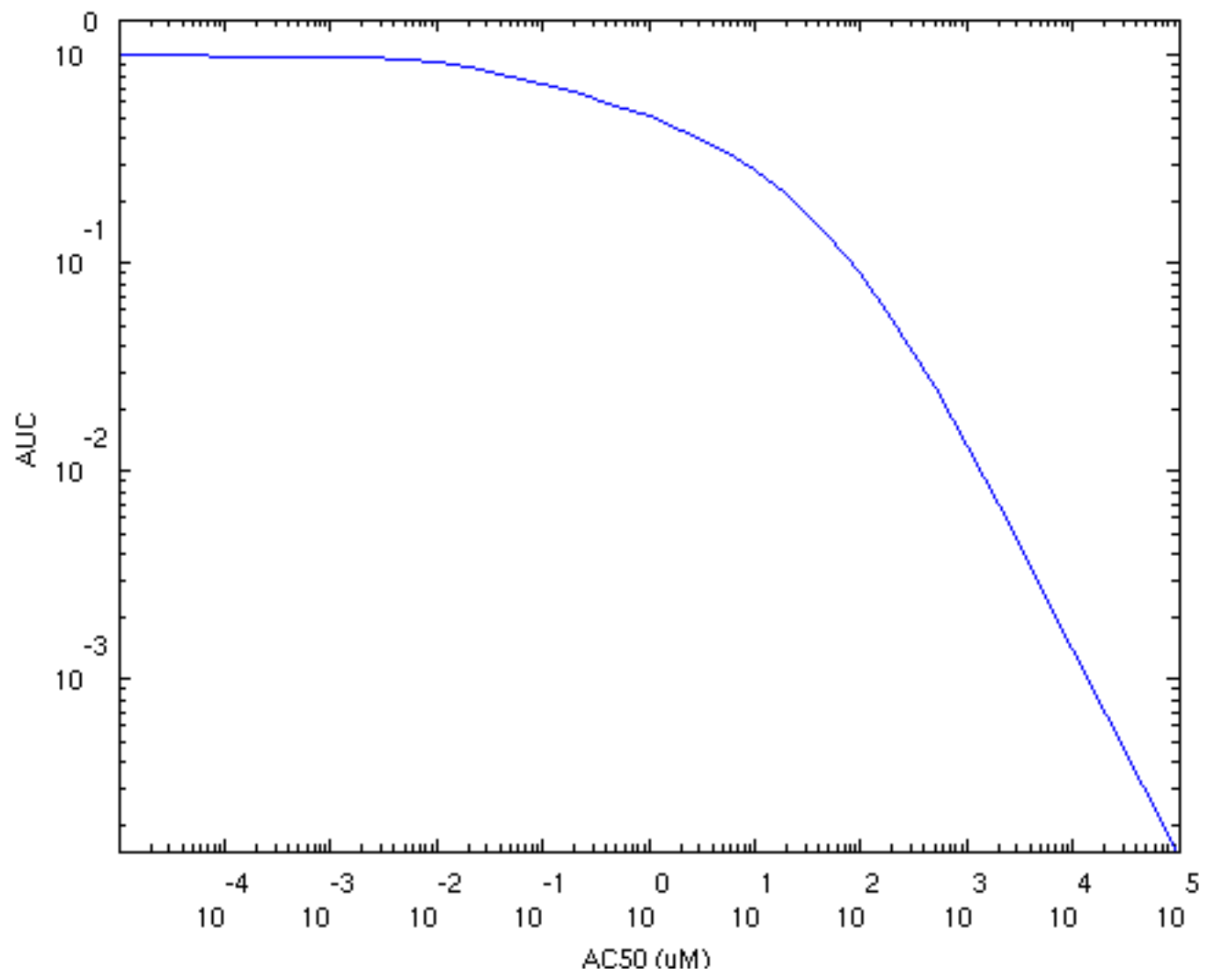

Figure S1. Plot of ER AUC scores vs $\mathbf{A C}_{50}$ values. Regression line demonstrating relationship between ER AUC scores and $\mathrm{AC}_{50}$ values. $\left(\mathrm{AC}_{50}=\right.$ activity concentration at half-maximal response). 


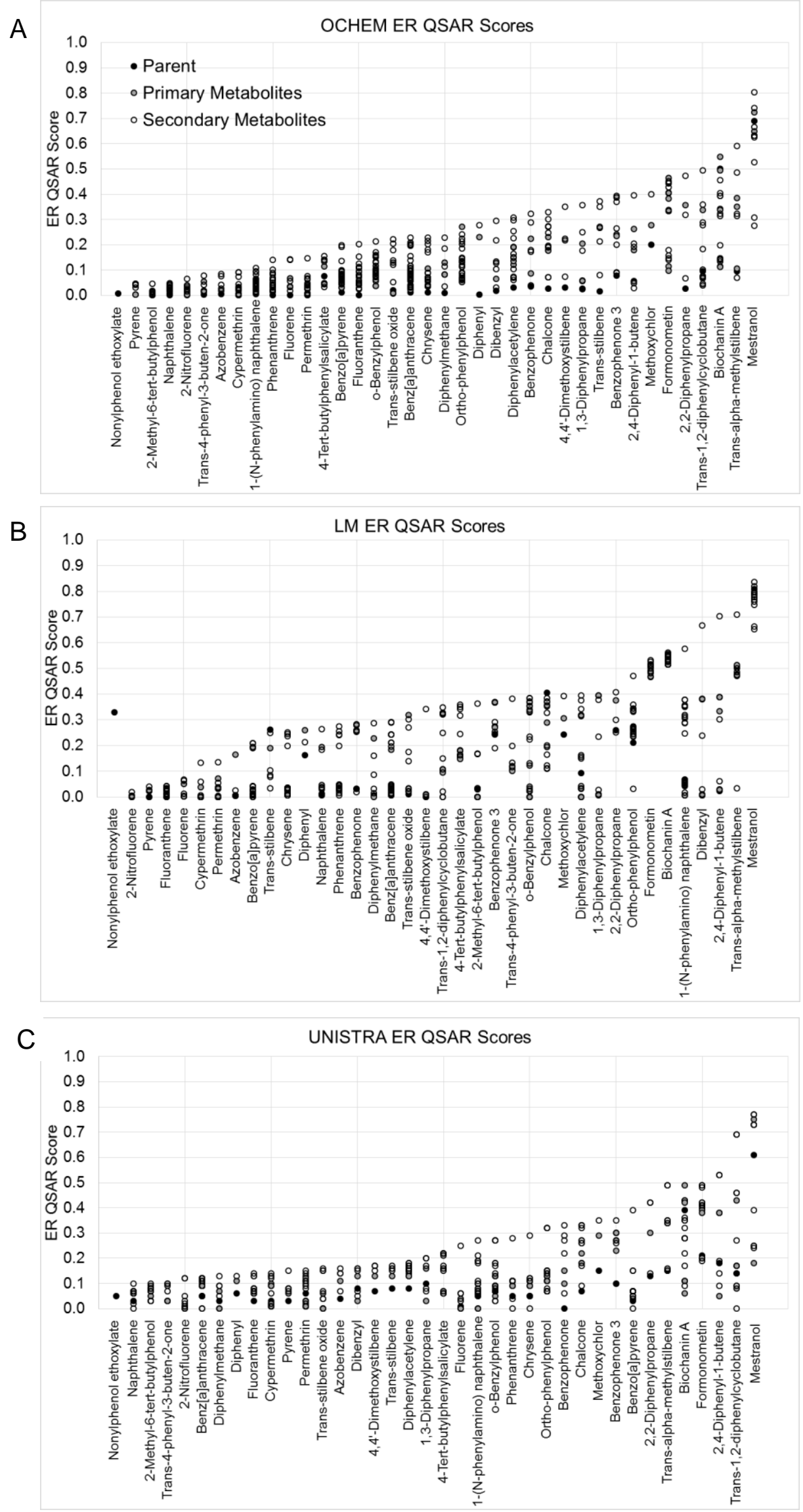


Figure S2. Estimation of estrogenic activity of parent chemicals and all predicted primary and secondary metabolites. ER QSAR scores of parent chemicals and all predicted primary and secondary metabolites are indicated for OCHEM (A), LM (C) and Unistra (E) ER QSAR models. Chemicals are ordered ascendingly based on the metabolite score (black circles - parent chemicals, gray circles - primary metabolites, white circles - secondary metabolites). No metabolites were generated for nonyphenol ethoxylate. 


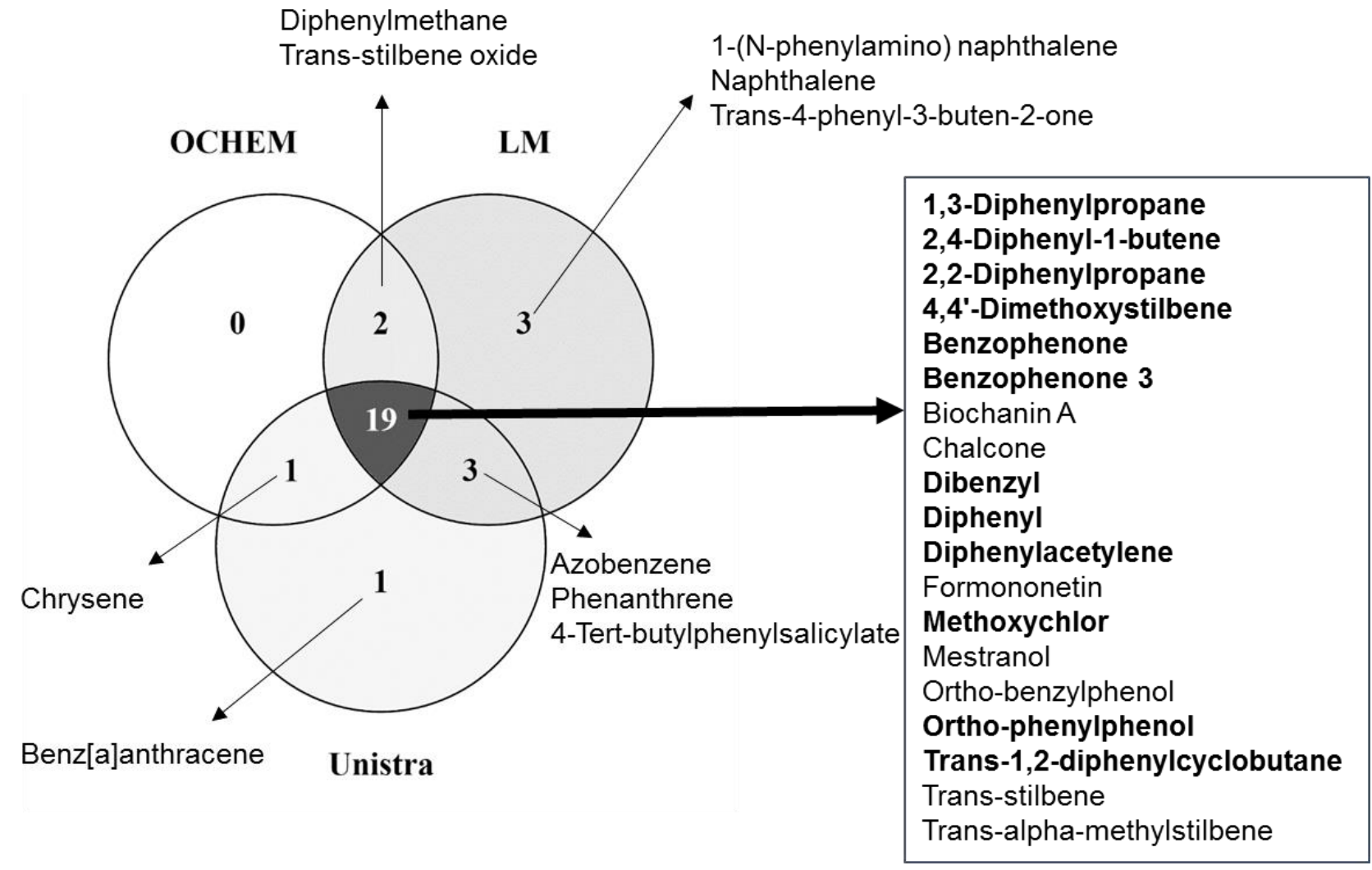

Figure S3. Parent chemicals predicted by the ER QSAR models to have metabolite scores $\geq 0.1$. A total of 19 parent chemicals were unanimously predicted by the three QSAR models to have metabolite scores $\geq 0.1$. Parent chemicals with metabolite scores $\geq 0.1$ and predicted to be bioactivated by the three QSAR models are indicated in the box in bold letters. 


\section{A}

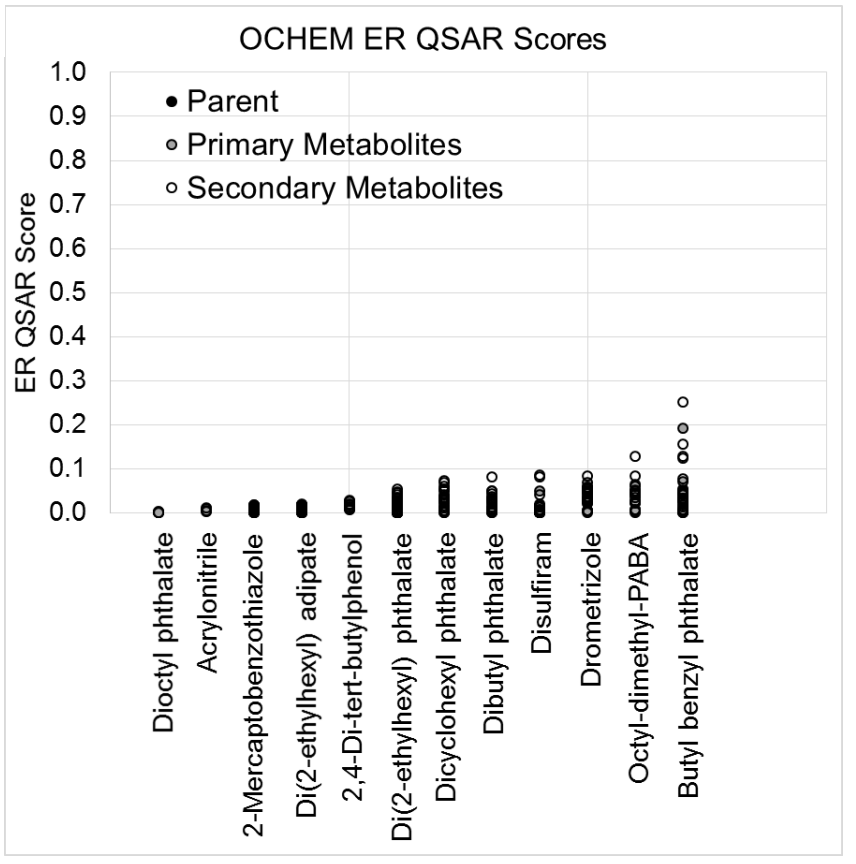

C

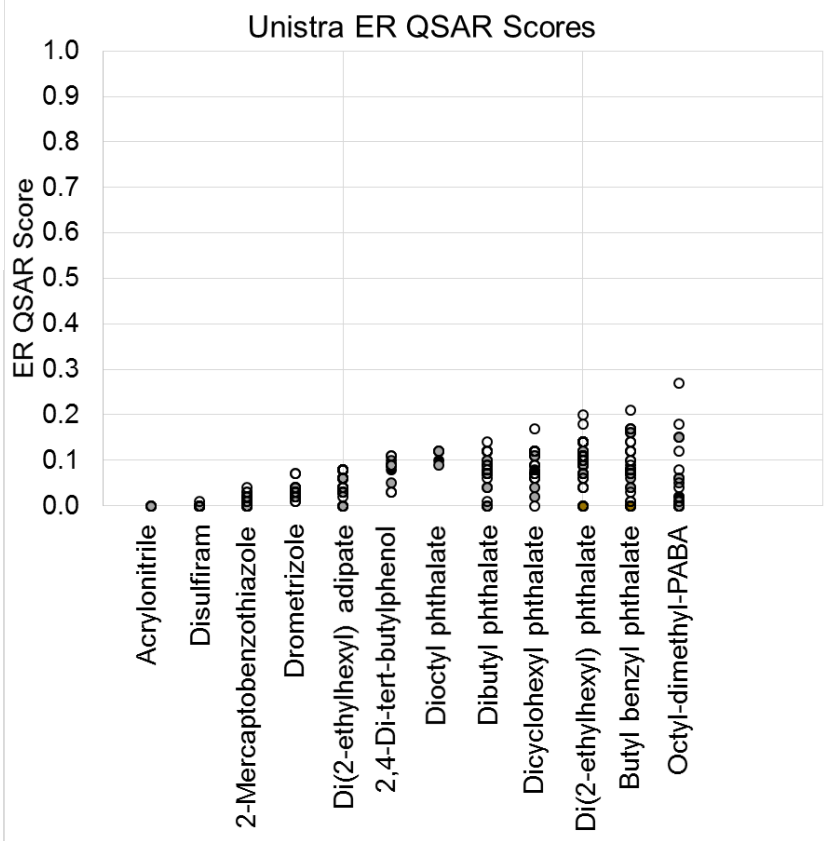

$\mathrm{B}$

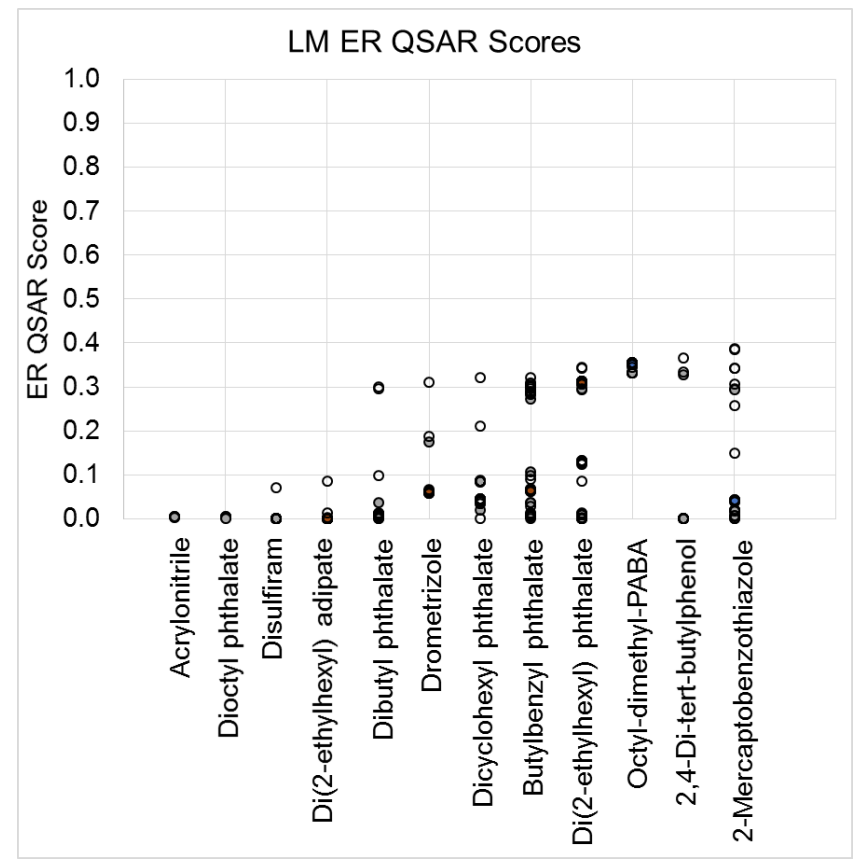

Figure S4. Estimation of estrogenic activity of parent chemicals and all predicted primary and secondary metabolites for chemicals not expected to undergo bioactivation. ER QSAR scores of parent chemicals and all predicted primary and secondary metabolites are indicated for OCHEM (A), LM (B) and Unistra (C) ER QSAR models. Chemicals are ordered ascendingly based on the metabolite score (black circles - parent chemicals, gray circles - primary metabolites, white circles - secondary metabolites). 


\section{References}

(1) Morohoshi, K., Yamamoto, H., Kamata, R., Shiraishi, F., Koda, T., and Morita, M. (2005) Estrogenic activity of 37 components of commercial sunscreen lotions evaluated by in vitro assays. Toxicol. In Vitro 19, 457-469.

(2) Ogawa, Y., Kawamura, Y., Wakui, C., Mutsuga, M., Nishimura, T., and Tanamoto, K. (2006) Estrogenic activities of chemicals related to food contact plastics and rubbers tested by the yeast two-hybrid assay. Food Addit. Contam. 23, 422-430.

(3) Kleinstreuer, N. C., Ceger, P. C., Allen, D. G., Strickland, J., Chang, X., Hamm, J. T., and Casey, W. M. (2016) A Curated Database of Rodent Uterotrophic Bioactivity. Environ. Health Perspect. 124, 556-562.

(4) Ohno, K., Azuma, Y., Date, K., Nakano, S., Kobayashi, T., Nagao, Y., and Yamada, T. (2003) Evaluation of styrene oligomers eluted from polystyrene for estrogenicity in estrogen receptor binding assay, reporter gene assay, and uterotrophic assay. Food Chem. Toxicol. 41, 131-141.

(5) Akahori, Y., Nakai, M., Yamasaki, K., Takatsuki, M., Shimohigashi, Y., and Ohtaki, M. (2008) Relationship between the results of in vitro receptor binding assay to human estrogen receptor alpha and in vivo uterotrophic assay: Comparative study with 65 selected chemicals. Toxicol. In Vitro 22, 225-231.

(6) Yamasaki, K., Takeyoshi, M., Sawaki, M., Imatanaka, N., Shinoda, K., and Takatsuki, M. (2003) Immature rat uterotrophic assay of 18 chemicals and Hershberger assay of 30 chemicals. Toxicology 183, 93-115.

(7) Ohta, R., Takagi, A., Ohmukai, H., Marumo, H., Ono, A., Matsushima, Y., Inoue, T., Ono, H., and Kanno, J. (2012) Ovariectomized mouse uterotrophic assay of 36 chemicals. J. Toxicol. Sci. 37, 879-889.

(8) Kummer, V., Maskova, J., Zraly, Z., Neca, J., Simeckova, P., Vondracek, J., and Machala, M. (2008) Estrogenic activity of environmental polycyclic aromatic hydrocarbons in uterus of immature Wistar rats. Toxicol. Lett. 180, 212-221.

(9) Fertuck, K. C., Matthews, J. B., and Zacharewski, T. R. (2001) Hydroxylated benzo[a]pyrene metabolites are responsible for in vitro estrogen receptor-mediated gene expression induced by benzo[a]pyrene, but do not elicit uterotrophic effects in vivo. Toxicol. Sci. 59, 231-240.

(10) Nakagawa, Y., and Tayama, K. (2001) Estrogenic potency of benzophenone and its metabolites in juvenile female rats. Arch. Toxicol. 75, 74-79.

(11) Koda, T., Umezu, T., Kamata, R., Morohoshi, K., Ohta, T., and Morita, M. (2005) Uterotrophic effects of benzophenone derivatives and a p-hydroxybenzoate used in ultraviolet screens. Environ. Res. 98, 40-45.

(12) Suzuki, T., Kitamura, S., Khota, R., Sugihara, K., Fujimoto, N., and Ohta, S. (2005) Estrogenic and antiandrogenic activities of 17 benzophenone derivatives used as UV stabilizers and sunscreens. Toxicol. Appl. Pharmacol. 203, 9-17.

(13) Yamasaki, K., Noda, S., Imatanaka, N., and Yakabe, Y. (2004) Comparative study of the uterotrophic potency of 14 chemicals in a uterotrophic assay and their receptor-binding affinity. Toxicol. Lett. 146, 111-120.

(14) Mu, H., Bai, Y. H., Wang, S. T., Zhu, Z. M., and Zhang, Y. W. (2009) Research on antioxidant effects and estrogenic effect of formononetin from Trifolium pratense (red clover). Phytomedicine 16, 314-319.

(15) U.S.EPA. (2015) EDSP: Weight of Evidence Analysis of Potential Interation with the Estrogen, Androgen OR Thyroid Pathways. https://www.epa.gov/sites/production/files/2015-06/documents/o-phenylphenol064103_2015-06-29_txr0057146.pdf.

(16) Sanoh, S., Kitamura, S., Sugihara, K., Kohta, R., Ohta, S., and Watanabe, H. (2006) Effects of stilbene and related compounds on reproductive organs in B6C3F1/Crj mouse. J. Health Sci. 52, 613-622. 